\title{
Second Derivative Free Eighteenth Order Convergent Method for Solving Non-Linear Equations
}

\author{
V.B. KUMAR VATTI ${ }^{1}$, RAMADEVI SRI ${ }^{1}$ and M.S. KUMAR MYLAPALLI ${ }^{2}$ \\ 1'Dept. of Engineering Mathematics, Andhra University, Visakhapatnam, India. \\ ${ }^{2}$ Dept. of Mathematics, Gitam University, Visakhapatnam, India.
}

\begin{abstract}
In this paper, the Eighteenth Order Convergent Method (EOCM) developed by Vatti et.al is considered and this method is further studied without the presence of second derivative. It is shown that this method has same efficiency index as that of EOCM. Several numerical examples are given to illustrate the efficiency and performance of the new method. AMS Subject Classification: 41A25, 65K05, 65H05.
\end{abstract}

\section{Introduction}

It is well known that a wide class of problems, which arises in diverse disciplines of mathematical and engineering science can be studied by the nonlinear equation of the form

$$
f(x)=0
$$

Where $f: D \subset R \rightarrow R$ is a scalar function on an open interval $D$ and $f(x)$ may be algebraic, transcendental or combined of both. The most widely used algorithm for solving (1.1) by the use of value of the function and its derivative is the well known quadratic convergent Newton's method (NM) given by

$$
x_{n+1}=x_{n}-\frac{f\left(x_{n}\right)}{f^{\prime}\left(x_{n}\right)} \underbrace{}_{(n=0,1,2, \ldots)}
$$

Article History

Received: 14 December 2017

Accepted: 25 Decmber 2017

\section{Keywords}

Iterative method, Nonlinear equation, Newton's method, Convergence analysis, Higher order convergence

starting with an initial guess $x_{0}$ which is in the vicinity of the exact root $x^{*}$. The efficiency index of Newton's method is $\sqrt[2]{2}=1.4142$.

The Extrapolated Newton's method (ENM) suggested by V.B.Kumar, Vatti et.al., ${ }^{11}$ which is developed by extrapolating Newton's method (1.2) introducing a parameter ' $\alpha_{\mathrm{n}}$ ' given by

$$
x_{n+1}=\left(1-\alpha_{n}\right) x_{n}+\alpha_{n}\left[x_{n}-\frac{f\left(x_{n}\right)}{f^{\prime}\left(x_{n}\right)}\right]
$$

\footnotetext{
CONTACT Dr. M. M. Sandeep Kumar manisandeepkumar.mylapalli@gitam.edu 9 Dept. of Mathematics, Gitam University, Visakhapatnam, India.

(C) 2017 The Author(s). Published by Techno Research Publishers

This is an $\mathbf{6}$ Open Access article licensed under a Creative Commons Attribution-NonCommercial-ShareAlike 4.0 International License (https://creativecommons.org/licenses/by-nc-sa/4.0/ ), which permits unrestricted NonCommercial use, distribution, and reproduction in any medium, provided the original work is properly cited.

To link to this article: http://dx.doi.org/10.13005/ojcst/10.04.19
} 
$x_{n+1}=x_{n}-\alpha_{n} \frac{f\left(x_{n}\right)}{f^{\prime}\left(x_{n}\right)}$

$$
(n=0,1,2, \ldots)
$$

Here the optimal choice for the parameter ' $\alpha_{n}$ ' is

$\alpha_{n}=\frac{2}{2-\rho_{n}}$

Where $\rho_{n}=\frac{f\left(x_{n}\right) f^{\prime \prime}\left(x_{n}\right)}{\left[f^{\prime}\left(x_{n}\right)\right]^{2}}$

Combining (1.3), (1.4) and (1.5), one can have

$$
x_{n+1}=x_{n}-\frac{f\left(x_{n}\right)}{f^{\prime}\left(x_{n}\right)} \cdot \frac{1}{\left[1-\frac{f\left(x_{n}\right) f^{\prime \prime}\left(x_{n}\right)}{2\left[f^{\prime}\left(x_{n}\right)\right]^{2}}\right]}{ }_{(n=0,1,2, \ldots)}
$$

Which is same as Halley's method having third order convergence which requires three functional evaluations. The efficiency index of this method is $\sqrt[3]{3}=1.4422$

A three step Predictor-corrector Newton's Halley method (PCNH) suggested by Mohammed and Hafiz $^{10}$, is given by:

For a given $\mathrm{x}_{0}$, compute $\mathrm{x}_{\mathrm{n}+1}$ by using

$$
\left.\begin{array}{l}
w_{n}=x_{n}-\frac{f\left(x_{n}\right)}{f^{\prime}\left(x_{n}\right)} \\
y_{n}=w_{n}-\frac{2 f\left(w_{n}\right) f^{\prime}\left(w_{n}\right)}{2\left[f^{\prime}\left(w_{n}\right)\right]^{2}-f\left(w_{n}\right) f^{\prime \prime}\left(w_{n}\right)} \\
x_{n+1}=y_{n}-\frac{f\left(y_{n}\right)}{f^{\prime}\left(y_{n}\right)}-\frac{\left[f\left(y_{n}\right)\right]^{2} f^{\prime \prime}\left(y_{n}\right)}{2\left[f^{\prime}\left(y_{n}\right)\right]^{3}} \\
(n=0,1,2, \ldots)
\end{array}\right\}
$$

This method has eighteenth order convergence and its efficiency index is $\sqrt[8]{18}=1.4352$.

The three step Eighteenth Order Extrapolated Newton's method (EOCM) developed by Vatti et.al., ${ }^{12}$ is given by:

For a given $x_{0}$, compute $x_{n+1}$ by the iterative schemes

$$
\left.\begin{array}{l}
w_{n}=x_{n}-\frac{f\left(x_{n}\right)}{f^{\prime}\left(x_{n}\right)} \\
y_{n}=w_{n}-\frac{f\left(w_{n}\right)}{f^{\prime}\left(w_{n}\right)}\left[\frac{1}{1-\hat{\rho} / 2}\right] \\
x_{n+1}=y_{n}-\frac{2 f\left(y_{n}\right)}{f^{\prime}\left(y_{n}\right)}\left[\frac{1}{1+\sqrt{1-2 \rho_{n}}}\right]
\end{array}\right\}
$$

Where $\hat{\rho}_{n}=\frac{f\left(w_{n}\right) f^{\prime \prime}\left(w_{n}\right)}{\left[f^{\prime}\left(w_{n}\right)\right]^{2}}$

and $\rho_{n}=\frac{f\left(y_{n}\right) f^{\prime \prime}\left(y_{n}\right)}{\left[f^{\prime}\left(y_{n}\right)\right]^{2}}$

This method has eighteenth order convergence and its efficiency index is $\sqrt[8]{18}=1.4352$.

In section 2, we consider the Second derivative free Eighteenth Order Extrapolated Newton's method and discuss the convergence criteria of this method in section 3. Few numerical examples are considered to show the superiority of this method in the concluding section.

\section{Second Derivative Free Eighteenth Order Convergent Method (Seocm)}

Considering the Eighteenth Order Extrapolated Newton's method (EOCM) (1.8) with (1.9) and (1.10), and expanding about, we obtain

$$
\begin{aligned}
& f\left(w_{n}\right)=f\left(x_{n}\right)+\left(y_{n}-x_{n}\right) f^{\prime}\left(x_{n}\right)+\frac{\left(y_{n}-x_{n}\right)^{2}}{2 !} f^{\prime \prime}\left(x_{n}\right) \\
& =f\left(x_{n}\right)+\left(\frac{-f\left(x_{n}\right)}{f^{\prime}\left(x_{n}\right)}\right) f^{\prime}\left(x_{n}\right)+\left(\frac{f^{2}\left(x_{n}\right)}{2 f^{\prime 2}\left(x_{n}\right)}\right) f^{\prime \prime}\left(x_{n}\right)
\end{aligned}
$$

on taking $w_{n}-x_{n}=\frac{-f\left(x_{n}\right)}{f^{\prime}\left(x_{n}\right)}$

in which case $\hat{\rho}_{n} \rightarrow 0$

Now,

$f\left(w_{n}\right)=\frac{f\left(x_{n}\right)}{2} \cdot \hat{\rho}_{n}$, where $\hat{\rho}_{n}=\frac{f\left(x_{n}\right) f^{\prime \prime}\left(x_{n}\right)}{\left[f^{\prime}\left(x_{n}\right)\right]^{2}}$, 
which gives

$$
\hat{\rho}_{n}=\frac{2 f\left(w_{n}\right)}{f\left(x_{n}\right)}
$$

Therefore, (1.9) takes the form

$$
\hat{\rho}_{n}=\frac{2 f\left(x_{n}-\frac{f\left(x_{n}\right)}{f^{\prime}\left(x_{n}\right)}\right)}{f\left(x_{n}\right)}
$$

Similarly, we can have

$$
\rho_{n}=\frac{2 f\left(y_{n}-\frac{f\left(y_{n}\right)}{f^{\prime}\left(y_{n}\right)}\right)}{f\left(y_{n}\right)}
$$

and rewriting equations (2.2), (2.3) in (1.8), we thus have the following algorithm:

\section{Algorithm 2.1}

For a given $x_{0}$, compute $x_{n+1}$ by the iterative schemes

$$
\left.\begin{array}{r}
w_{n}=x_{n}-\frac{f\left(x_{n}\right)}{f^{\prime}\left(x_{n}\right)} \\
y_{n}=w_{n}-\frac{f\left(w_{n}\right)}{f^{\prime}\left(w_{n}\right)}\left[\frac{1}{1-\hat{\rho} / 2}\right] \\
x_{n+1}=y_{n}-\frac{2 f\left(y_{n}\right)}{f^{\prime}\left(y_{n}\right)}\left[\frac{1}{1+\sqrt{1-2 \rho_{n}}}\right] \\
(n=0,1,2, \ldots)
\end{array}\right\}
$$

Where $\hat{\rho}_{n}$ and $\rho_{n}$ are as given in (2.2) and (2.3).

This algorithm can be called as Second derivative free Eighteenth Order Extrapolated Newton's method (SEOCM) and it requires eight functional evaluations.

\section{Convergence Criteria}

\section{Theorem 3.1}

Let $x^{*} \in D$ be a single zero of a sufficiently differentiable function $f: D \subset R \rightarrow R$ for an open interval $D$ and let $x_{0}$ be in the vicinity of $x^{*}$, then the algorithm (2.1) has eighteenth order convergence.

\section{Proof}

Let be a single zero of (1.1) and

$x^{*}=x_{n}+e_{n}$

Then, $f\left(x^{\star}\right)=0$

If $x_{n}$ be the $n^{\text {th }}$ approximate to the root of (1.1), then expanding $f\left(x_{n}\right)$ about $x^{*}$ using Taylor's expansion, we have

$f\left(x_{n}\right)=f\left(x^{*}\right)+e_{n} f^{\prime}\left(x^{*}\right)+\frac{e_{n}^{2}}{2 !} f^{\prime \prime}\left(x^{*}\right)+\frac{e_{n}^{3}}{3 !} f^{\prime \prime \prime}\left(x^{*}\right)+\ldots$

$f\left(x_{n}\right)=f^{\prime}\left(x^{*}\right)\left[e_{n}+\frac{1}{2 !} \frac{f^{\prime \prime}\left(x^{*}\right)}{f^{\prime}\left(x^{*}\right)} e_{n}{ }^{2}+\frac{1}{3 !} \frac{f^{\prime \prime \prime}\left(x^{*}\right)}{f^{\prime}\left(x^{*}\right)} e_{n}{ }^{3}+\frac{1}{4 !} \frac{f^{\prime v}\left(x^{*}\right)}{f^{\prime}\left(x^{*}\right)} e_{n}{ }^{4}+\ldots\right]$

$f\left(x_{n}\right)=f^{\prime}\left(x^{*}\right)\left[e_{n}+c_{2} e_{n}{ }^{2}+c_{3} e_{n}{ }^{3}+c_{4} e_{n}{ }^{4}+\ldots\right]$

Where $c_{j}=\frac{1}{j !} \cdot \frac{f^{j}\left(x^{*}\right)}{f^{\prime}\left(x^{*}\right)}, \quad(j=2,3,4, \ldots)$

And,

$f^{\prime}\left(x_{n}\right)=f^{\prime}\left(x^{*}\right)\left[1+2 c_{2} e_{n}+3 c_{3} e_{n}{ }^{2}+4 c_{4} e_{n}^{3}+\ldots\right]$

Now,

$\frac{f\left(x_{n}\right)}{f^{\prime}\left(x_{n}\right)}=e_{n}-c_{2} e_{n}^{2}-\left(2 c_{3}-2 c_{2}^{2}\right) e_{n}^{3}-\left(3 c_{4}-7 c_{2} c_{3}+4 c_{2}^{3}\right) e_{n}^{4}+o\left(e_{n}^{5}\right)$

From (2.4), (3.1) and (3.5), we have

$$
\begin{array}{r}
w_{n}=x^{*}+c_{2} e_{n}^{2}+\left(2 c_{3}-2 c_{2}{ }^{2}\right) e_{n}^{3}+\left(3 c_{4}-7 c_{2} c_{3}+4 c_{2}{ }^{3}\right) e_{n}{ }^{4}+o\left(e_{n}^{5}\right) \\
\text { Let } W=w_{n}-x^{*}
\end{array}
$$

Then, expanding $f\left(w_{n}\right), f^{\prime}\left(w_{n}\right), f^{\prime \prime}\left(w_{n}\right)$ about by using (3.6), we obtain

$f\left(w_{n}\right)=f\left(x^{*}\right)+\left(w_{n}-x^{*}\right) f^{\prime}\left(x^{*}\right)+\frac{\left(w_{n}-x^{*}\right)^{2}}{2 !} f^{\prime \prime}\left(x^{*}\right)+\frac{\left(w_{n}-x^{*}\right)^{3}}{3 !} f^{\prime \prime \prime}\left(x^{*}\right)+\ldots$ 
$=f^{\prime}\left(x^{*}\right)\left[W+c_{2} W^{2}+c_{3} W^{3}+c_{4} W^{4}+\ldots\right]$

$f^{\prime}\left(w_{n}\right)=f^{\prime}\left(x^{*}\right)+\left(w_{n}-x^{*}\right) f^{\prime \prime}\left(x^{*}\right)+\frac{\left(w_{n}-x^{*}\right)^{2}}{2 !} f^{\prime \prime \prime}\left(x^{*}\right)+\ldots$

$=f^{\prime}\left(x^{*}\right)\left[1+2 c_{2} W+3 c_{3} W^{2}+4 c_{4} W^{3}+\ldots\right]$

$\frac{f\left(w_{n}\right)}{f^{\prime}\left(w_{n}\right)}=W-c_{2} W^{2}-\left(2 c_{3}-2 c_{2}^{2}\right) W^{3}-\left(3 c_{4}-7 c_{2} c_{3}+4 c_{2}^{3}\right) W^{4}+o\left(e_{n}^{5}\right)$

$w_{n}-\frac{f\left(w_{n}\right)}{f^{\prime}\left(w_{n}\right)}=x^{*}+c_{2} W^{2}+\left(2 c_{3}-2 c_{2}^{2}\right) W^{3}+\left(3 c_{4}-7 c_{2} c_{3}+4 c_{2}^{3}\right) W^{4}+o\left(e_{n}^{5}\right)$

$f\left(w_{n}-\frac{f\left(w_{n}\right)}{f^{\prime}\left(w_{n}\right)}\right)=f^{\prime}\left(x^{*}\right)\left[c_{2} W^{2}+\left(2 c_{3}-2 c_{2}^{2}\right) W^{3}+\left(3 c_{4}-7 c_{2} c_{3}+4 c_{2}^{3}\right) W^{4}+o\left(e_{n}^{5}\right)\right]$

and $\hat{\rho}_{n}=\frac{2 f\left(w_{n}-\frac{f\left(w_{n}\right)}{f^{\prime}\left(w_{n}\right)}\right)}{f\left(w_{n}\right)}$

$=2\left[c_{2} W+\left(2 c_{3}-3 c_{2}^{2}\right) W^{2}+\left(3 c_{4}-10 c_{2} c_{3}+7 c_{2}^{3}\right) W^{3}+\left(9 c_{2}^{2} c_{3}-4 c_{2} c_{4}-5 c_{2}^{4}-2\left(c_{3}-c_{2}^{2}\right)^{2}\right) W^{4}+0\left(e_{n}^{5}\right)\right]$

$=2\left[S_{1} W+S_{2} W^{2}+S_{3} W^{3}+S_{4} W^{4}+\ldots\right]$

where,

$S_{1}=c_{2}, S_{2}=2 c_{3}-3 c_{2}^{2}, S_{3}=3 c_{4}-10 c_{2} c_{3}+7 c_{2}^{3}$

$S_{4}=9 c_{2}{ }^{2} c_{3}-4 c_{2} c_{4}-5 c_{2}{ }^{4}-2\left(c_{3}-c_{2}{ }^{2}\right)^{2}$

Now,

$\left[1-\frac{\hat{\rho}_{n}}{2}\right]^{-1}=\left[1-\left(S_{1} W+S_{2} W^{2}+S_{3} W^{3}+S_{4} W^{4}+\ldots\right)\right]^{-1}$

$=1+S_{1} W+\left(S_{1}^{2}+S_{2}\right) W^{2}+\left(2 S_{1} S_{2}+S_{1}^{3}+S_{3}\right) W^{3}$

$+\left(S_{4}+S_{2}^{2}+2 S_{1} S_{3}+3 S_{1}^{2} S_{2}+S_{1}^{4}\right) W^{4}+\ldots$
Multiplying (3.10) with (3.14), we obtain

$\frac{f\left(w_{n}\right)}{f^{\prime}\left(w_{n}\right)}\left[1-\frac{\hat{p}_{n}}{2}\right]^{-1}=\left[W-c_{2} \mathbb{W}^{2}-\left(2 c_{3}-2 c_{2}^{2}\right) W^{3}-\left(3 c_{4}-7 c_{2} c_{3}+4 c_{2}^{3}\right) W^{4}+o\left(e_{n}^{5}\right)\right]$.

$\left[1+S_{1} W+\left(S_{1}^{2}+S_{2}\right) W^{2}+\left(2 S_{1} S_{2}+S_{1}^{3}+S_{3}\right) W^{3}+\left(S_{4}+S_{2}^{2}+2 S_{1} S_{3}+3 S_{1}^{2} S_{2}+S_{1}^{4}\right) W^{4}+\ldots\right]$

$=W+\left(-c_{2}^{2}\right) W^{3}+o\left(e_{n}^{4}\right)$

Combining (3.6) to (3.15) and from (2.4), we obtain

$$
y_{n}=x^{*}+T
$$

where, $T=c_{2}{ }^{3} e_{n}{ }^{6}$

Now, expanding $f\left(y_{n}\right), f^{\prime}\left(y_{n}\right)$ about $x^{*}$ by using (3.16), we obtain

$f\left(y_{n}\right)=f^{\prime}\left(x^{*}\right)\left[T+c_{2} T^{2}+c_{3} T^{3}+c_{4} T^{4}+\ldots\right]$

$f^{\prime}\left(y_{n}\right)=f^{\prime}\left(x^{*}\right)\left[1+2 c_{2} T+3 c_{3} T^{2}+4 c_{4} T^{3}+5 c_{5} T^{4}+\ldots\right]$

Now,

$\frac{f\left(y_{n}\right)}{f^{\prime}\left(y_{n}\right)}=T-c_{2} T^{2}-\left(2 c_{3}-2 c_{2}^{2}\right) T^{3}-\left(3 c_{4}-7 c_{2} c_{3}+4 c_{2}^{3}\right) T^{4}+o\left(e_{n}^{5}\right)$

$y_{n}-\frac{f\left(y_{n}\right)}{f^{\prime}\left(y_{n}\right)}=x^{*}+c_{2} T^{2}+\left(2 c_{3}-2 c_{2}{ }^{2}\right) T^{3}+\left(3 c_{4}-7 c_{2} c_{3}+4 c_{2}{ }^{3}\right) T^{4}+o\left(e_{n}^{5}\right)$

$f\left(y_{n}-\frac{f\left(y_{n}\right)}{f^{\prime}\left(y_{n}\right)}\right)=f^{\prime}\left(x^{*}\right)\left[c_{2} T^{2}+\left(2 c_{3}-2 c_{2}{ }^{2}\right) T^{3}+\left(3 c_{4}-7 c_{2} c_{3}+4 c_{2}{ }^{3}\right) T^{4}+o\left(e_{n}{ }^{5}\right)\right]$

and $\rho_{n}=\frac{2 f\left(y_{n}-\frac{f\left(y_{n}\right)}{f^{\prime}\left(y_{n}\right)}\right)}{f\left(y_{n}\right)}$

$$
\begin{aligned}
& =2\left[c_{2} T+\left(2 c_{3}-3 c_{2}{ }^{2}\right) T^{2}+\left(3 c_{4}-10 c_{2} c_{3}+7 c_{2}{ }^{3}\right) T^{3}\right. \\
& \left.\left(9 c_{2}{ }^{2} c_{3}-4 c_{2} c_{4}-5 c_{2}{ }^{4}-2\left(c_{3}-c_{2}{ }^{2}\right)^{2}\right) T^{4}+o\left(e_{n}{ }^{5}\right)\right]
\end{aligned}
$$


$=2\left[P_{1} T+P_{2} T^{2}+P_{3} T^{3}+P_{4} T^{4}+\ldots\right]$

where

$P_{1}=c_{2}, P_{2}=2 c_{3}-3 c_{2}^{2}, P_{3}=3 c_{4}-10 c_{2} c_{3}+7 c_{2}^{3}, P_{4}=$ $9 \mathrm{c}_{2}{ }^{2} \mathrm{C}_{3}-4 \mathrm{c}_{2} \mathrm{c}_{4}+5 \mathrm{c}_{2}^{4}-2\left(\mathrm{c}_{3}-\mathrm{c}_{2}\right)^{2}$

Now,

$$
\begin{aligned}
& \sqrt{1-2 \rho_{n}}=\left[1-2 P_{1} T+2\left(-\frac{P_{1}^{2}}{2}-P_{2}\right) T^{2}+2\left(-P_{3}-P_{1} P_{2}-\frac{P_{1}^{3}}{2}\right) T^{3}\right. \\
& \left.2\left(-P_{4}-\frac{P_{2}^{2}}{2}-P_{1} P_{3}-\frac{3}{2} P_{1}^{2} P_{2}-\frac{5}{8} P_{1}^{4}\right) T^{4}+\ldots\right]
\end{aligned}
$$

and,

$1+\sqrt{1-2 \rho_{n}}=2\left[1-P_{1} T-\left(\frac{P_{1}^{2}}{2}+P_{2}\right) T^{2}-\left(P_{3}+P_{1} P_{2}+\frac{P_{1}^{3}}{2}\right) T^{3}\right.$

$\left.-\left(P_{4}+\frac{P_{2}^{2}}{2}+P_{1} P_{3}+\frac{3}{2} P_{1}^{2} P_{2}+\frac{5}{8} P_{1}^{4}\right) T^{4}+\ldots\right]$

$1+\sqrt{1-2 \rho_{n}}=2\left[1+M_{1} T+M_{2} T^{2}+M_{3} T^{3}+M_{4} T^{4}+\ldots\right]$

where,

$$
\begin{aligned}
& M_{1}=-c_{2}, \quad M_{2}=\frac{5}{2} c_{2}{ }^{2}-2 c_{3}, \quad M_{3}=-\frac{9}{2} c_{2}{ }^{3}+8 c_{2} c_{3}-3 c_{4}, \\
& M_{4}=8 c_{2}{ }^{2} c_{3}-\frac{37}{8} c_{2}{ }^{4}+c_{2} c_{4}-4 c_{3}{ }^{2}
\end{aligned}
$$

Now again,

$$
\begin{aligned}
& {\left[1+\sqrt{1-2 \rho_{n}}\right]^{-1}=\frac{1}{2}\left[1-M_{1} T+\left(M_{1}^{2}-M_{2}\right) T^{2}+\left(2 M_{1} M_{2}-M_{3}-M_{1}^{3}\right) T^{3}\right.} \\
& \left.+\left(M_{2}^{2}+2 M_{1} M_{4}-M_{4}-3 M_{1}^{2} M_{2}+M_{1}^{4}\right) T^{4}+\ldots\right] \\
& =\frac{1}{2}\left[1+N_{1} T+N_{2} T^{2}+N_{3} T^{3}+N_{4} T^{4}+\ldots\right]
\end{aligned}
$$

where,

$$
\begin{aligned}
& N_{1}=c_{2}, N_{2}=-\frac{3}{2} c_{2}{ }^{2}+2 c_{3}, N_{3}=-\frac{3}{2} c_{2}{ }^{3}-4 c_{2} c_{3} \\
& +3 c_{4}, N_{4}=-28 c_{2}{ }^{2} c_{3}+\frac{107}{8} c_{2}{ }^{4}+5 c_{2} c_{4}+8 c_{3}{ }^{2}
\end{aligned}
$$

From (3.20) and (3.26), we obtain

$$
\begin{aligned}
& 2 \cdot \frac{f\left(y_{n}\right)}{f^{\prime}\left(y_{n}\right)} \cdot\left[1+\sqrt{1-2 \hat{\rho}_{n}}\right]^{-1}=\left[T+\left[N_{1}-c_{2}\right] T^{2}+\left[N_{2}-N_{1} c_{2}+2 c_{2}{ }^{2}-2 c_{3}\right] T^{3}\right. \\
& \left.+\left[N_{3}-N_{2} c_{2}+N_{1}\left(2 c_{2}{ }^{2}-2 c_{3}\right)+7 c_{2} c_{3}-4 c_{2}{ }^{3}-3 c_{4}\right] T_{n}{ }^{4}+o\left(T^{5}\right)\right] \\
& =\left[T+[0] T^{2}+\left[-\frac{1}{2} c_{2}{ }^{2}\right] T^{3}+\left[N_{3}-N_{2} c_{2}+N_{1}\left(2 c_{2}{ }^{2}-2 c_{3}\right)+7 c_{2} c_{3}-4 c_{2}{ }^{3}-3 c_{4}\right] T_{n}{ }^{4}+o\left(T^{5}\right)\right]
\end{aligned}
$$

Combining (3.18) to (3.27) and from (2.4), we obtain

$$
\begin{aligned}
& x_{n+1}=\left[x^{*}+T\right]-\left[T-\frac{1}{2} c_{2}{ }^{2} T^{3}+\ldots\right] \\
& =x^{*}+\frac{1}{2} c_{2}{ }^{2} T^{3}+\ldots \\
& =x^{*}+\frac{1}{2} c_{2}{ }^{2}\left[c_{2}{ }^{3} e_{n}{ }^{6}\right]^{3}+\ldots
\end{aligned}
$$

i.e., $e_{n+1}=\frac{1}{2} c_{2}{ }^{11} e_{n}{ }^{18}+o\left(e_{n}^{19}\right) \ldots$

Hence, we have $e_{n+1}$ a $e_{n}^{18}$. Therefore, the algorithm (2.1) has eighteenth order convergence and its efficiency index is which is same as that of the method (1.7) and (1.8).

\section{Numerical Examples}

We consider the same examples considered by Mohammed and $\mathrm{Hafiz}^{10}$ and V.B.Kumar, Vatti et.al., ${ }^{13}$ and compared EOCM with NM and PCNH methods. The computations are carried out by using mpmath-PYTHON software programming and comparison of number of iterations for these methods are obtained such that $\left|x_{n+1}-x_{n}\right|<10^{-201}$ and $\left|f\left(x_{n+1}\right)\right|<10^{-201}$. 
Table 1: Comparison of different methods

\begin{tabular}{|c|c|c|c|c|c|}
\hline Method & $\begin{array}{l}\text { Initial Guess } \\
\mathrm{x}_{0}\end{array}$ & $\begin{array}{l}\text { The equation } f(x)=0 \text { and its } \\
\text { root by respective methods }\end{array}$ & $\begin{array}{l}\text { No. of } \\
\text { iterations }\end{array}$ & $\left|x_{n+1}-x_{n}\right|$ & $\left|f\left(x_{n+1}\right)\right|$ \\
\hline & 1 & $f(x)=x^{3}+4 x^{2}-10$ & & & \\
\hline NM & & 1.365230013414096845760806828 & 10 & 3.43 E-200 & $-5.75 E-199$ \\
\hline $\mathrm{PCNH}$ & & 1.365230013414096845760806828 & 5 & -3.27E-201 & $-5.75 E-199$ \\
\hline EOCM & & 1.365230013414096845760806828 & 3 & $-3.27 E-201$ & 1.07E-198 \\
\hline SEOCM & 1.3 & $\begin{array}{l}1.365230013414096845760806828 \\
f(x)=\sin ^{2} x-x^{2}+1\end{array}$ & 3 & $-9.8 E-200$ & $-5.75 E-199$ \\
\hline NM & & 1.404491648215341226035086817 & 9 & 6.86E-200 & 1.72E-199 \\
\hline $\mathrm{PCNH}$ & & 1.404491648215341226035086817 & 4 & $-6.53 E-201$ & -7.67E-200 \\
\hline EOCM & & 1.404491648215341226035086817 & 3 & 9.31E-200 & -7.67E-200 \\
\hline SEOCM & 1.7 & $\begin{array}{l}1.404491648215341226035086817 \\
f(x)=\cos x-x\end{array}$ & 3 & -7.67E-200 & 3.14E-199 \\
\hline NM & & 0.739085133215160641655312087 & 9 & 1.63E-201 & 2.45E-201 \\
\hline NM & & 0.739085133215160641655312087 & 4 & $-4.9 \mathrm{E}-201$ & 2.45E-201 \\
\hline EOCM & & 0.739085133215160641655312087 & 3 & $-4.9 \mathrm{E}-201$ & 2.45E-201 \\
\hline SEOCM & 2 & $\begin{array}{l}0.739085133215160641655312087 \\
f(x)=x^{3}-10\end{array}$ & 3 & 8.16E-202 & $-8.16 \mathrm{E}-202$ \\
\hline NM & & 2.154434690031883721759293566 & 9 & 1.63E-200 & $-2.1 \mathrm{E}-199$ \\
\hline $\mathrm{PCNH}$ & & 2.154434690031883721759293566 & 4 & $-4.9 E-200$ & $-2.1 \mathrm{E}-199$ \\
\hline EOCM & & 2.154434690031883721759293566 & 3 & $-4.9 E-200$ & $-2.1 E-199$ \\
\hline SEOCM & & 2.154434690031883721759293566 & 3 & -3.27E-201 & $-2.6 E-200$ \\
\hline
\end{tabular}

It is evident from these tabulated values that SEOCM is superior to the methods (1.2) and (1.7) considering the number of iterations and accuracy and the rate at which SEOCM converged and the convergence rate is almost same as that of the method (1.8). Of these methods, SEOCM is free from second derivatives.

\section{References}

$1 \quad$ I.K. Argyros, S.K. Khattri, An improved semi local convergence analysis for the Chebyshev method, Journal of Applied Mathematics and computing, 42, No-s: 1, 2 (2013), 509-528, doi: 10.1007/s12190-013-0647-3.

2 M.A. Hafiz, Solving nonlinear equations using steffensen-type methods with optimal order of convergence, Palestine Journal of Mathematics, 3, No: 1 (2014), 113-119.

3 M.A. Hafiz, A new combined bracketing method for solving nonlinear equations, Journal of Mathematical and Computational Science, 3, No.1 (2013)..

4 M.A. Hafiz, S.M.H. Al-Goria, Solving nonlinear equations using a new tenth-and seventhorder methods free from second derivative,
International Journal of Differential Equations and Applications, 12, No. 3 (2013), 169183,2013, doi: 10.12732/ijdea.v12i3.1344.

5 S.K. Khattri, Torgrim Log, Constructing thirdorder derivative-free iterative methods, Int. J. Comput. Math., 88, No. 7 (2011), 1509-1518, doi: 10.1080/00207160.2010520705.

$6 \quad$ S.K. Khattri, Quadrature based optimal iterative methods with applications in high precision computing, Numer. Math. Theor. Meth. Appl., 5 (2012), 592-601.

7 S.K. Khattri, Trond Steihaug, Algorithm for forming derivative-free optimal methods, , Numerical Algorithms, (2013), doi: 10.1007/ s11075-013-9715-x.

8 Mohamed S.M. Bahgat, M.A. Hafiz, New 
two-step predictor-corrector method with ninth order convergence for solving nonlinear equations, Journal of Advances in Mathematics , 2 (2013), 432-437.

9 Khalida Inayat Noor, Muhammad Aslam Noor, Shaher Momani, Modified Householder iterative methods for nonlinear equations", Applied Mathematics and Computation, 190 (2007), 1534-1539, doi: 10.1016/j. amc.2007.02.036.

10

Mohamed S.M. Bahgat, M.A. Hafiz, Threestep iterative method with eighteenth order convergence for solving nonlinear equations, Int. Journal of Pure and Applied Mathematics, Vol. 93, No. 1, 2014, 85-94.
11 Vatti V.B.Kumar, Mylapalli M. S. Kumar, Katragadda A. Kumari,Extrapolated NewtonRaphson Method, Math. Educ. 43, No. 1, 103-108 (2009).

12 Vatti V. B. K, Sri R, Mylapalli M. S. K., Eighteenth Order Convergent Method for Solving Non-Linear Equations. Orient.J. Comp. Sci. and Technol;10(1), 144-150 (2017).

13 Vatti V. B. K, Ramadevi Sri, Mylapalli M. S. K., Two Step Extrapolated Newton's Method with High Efficiency Index, Journal of Advanced Research in Dynamical and Control Systems; 9(5), 08-15 (2017). 